\title{
Knowledge on postnatal care among postpartum mothers during discharge in maternity hospitals in Asmara: a cross- sectional study
}

Ghirmay Ghebreigziabher Beraki ${ }^{1 *}$ D, Eyasu H. Tesfamariam², Amanuel Gebremichael ${ }^{1}$, Berhanemeskel Yohannes ${ }^{1}$, Kessete Haile ${ }^{1}$, Shewit Tewelde ${ }^{1}$ and Simret Goitom ${ }^{1}$

\begin{abstract}
Background: The early postnatal period is a dangerous time for both mother and baby where morbidity and mortality are highly prevalent if proper care is not done. Post natal care (PNC) knowledge has significant role in reducing such complications. In this study, the knowledge of postpartum mothers on PNC and its determinants were determined.

Methods: A cross-sectional quantitative study was conducted in postpartum mothers (PpM) who attended all maternal delivery services in Asmara. Data was collected by a structured questionnaire. All $(n=250)$ PpM who gave birth in December, 2017 were included in the study. Independent samples t-test and one way ANOVA were used to compare the scores in knowledge across categories of background characteristics using SPSS. Bonferroni post-hoc test was performed for variables that were found to be significant while using ANOVA tool. P-values less than 0.05 were considered as significant.

Results: The percentage of PpM who cited vaginal bleeding, as a maternal danger sign, and fever, as a baby danger sign, were 83.2 and 58.8\%, respectively. The majority (96\%) of PpM responded the correct answer on where to go if they note any danger signs. In addition, more than nine tenth of PpM correctly identified injectable contraceptives (92.7\%) and oral contraceptive (91.5\%). The percentages of knowledge in recognizing the necessary nutrients ranged from $87.6 \%$ for carbohydrates to $46 \%$ for minerals. The percentages of correct knowledge regarding first baby bath, frequency of breast feeding, umbilical care, duration of exclusive breast feeding, need and purpose of vaccine were 40.1, 81.9, 77.4, 94.8, and 99.2\% respectively. The mean PNC knowledge score was 24.89/60. The score of knowledge on postnatal care was found to significantly differ across the categories of residence $(p<0.001)$ and ethnicity $(p=0.015)$. An increasing trend of knowledge score was observed with increase in age group $(p<0.001)$, educational level $(p=$ $0.021)$, gravida $(p<0.001)$ and para $(p<0.001)$.

Conclusion: Considerable gaps in knowledge regarding postnatal care among postpartum mothers were evident. Special attention should be laid on rural residents, single/living together, junior/below in educational level, primigravida/para, non-Tigrigna ethnicity, and 17 to 25 years old mothers.
\end{abstract}

Keywords: Knowledge, Postpartum mothers, Postnatal care

\footnotetext{
* Correspondence: gberaki83@gmail.com

${ }^{1}$ Department of Nursing, Orotta College of Medicine and Health Sciences,

Asmara, Eritrea

Full list of author information is available at the end of the article
}

(c) The Author(s). 2020 Open Access This article is distributed under the terms of the Creative Commons Attribution 4.0 International License (http://creativecommons.org/licenses/by/4.0/), which permits unrestricted use, distribution, and reproduction in any medium, provided you give appropriate credit to the original author(s) and the source, provide a link to the Creative Commons license, and indicate if changes were made. The Creative Commons Public Domain Dedication waiver (http://creativecommons.org/publicdomain/zero/1.0/) applies to the data made available in this article, unless otherwise stated. 


\section{Background}

Post-natal care refers to issues pertaining to the mother and the baby from birth up to 6 weeks [1]. The goal of care during the early postnatal period is to promote the physical well-being of both mother and baby, as well as support the developing relationship between the baby and his or her parents and family. In addition, it can also support the development of infant feeding skills and strengthen the mother's knowledge and confidence in her and her baby's health and well-being. Accordingly, postnatal care knowledge enables mothers to develop parenting skills to fulfill their mothering role within their particular family [2].

Lack of appropriate postnatal care sometimes may result in death or disability of the mother and/or newborn [3]. Worldwide, nearly 600,000 mothers between the ages of 15-49 years die every year due to complications arising from pregnancy and childbirth. Hence, maternal death occurs almost every minute of every year, out of which $99 \%$ are in the developing countries [3]. Around two thirds of maternal and newborn deaths occur in the early postpartum period in developing countries and most of them in sub-Saharan Africa $[1,4]$. Almost half of postnatal maternal deaths occur within the first $24 \mathrm{~h}$ and $66 \%$ occur during the first week [1, 5]. In 2013, 2.8 million newborns died in their first month of life, from which 1 million died on the first day [5].

There has been great emphasis on skilled attendant delivery and efforts have been made to improve PNC guidelines globally and nationally [1]. In sub-Saharan Africa, $48 \%$ of women give birth with the assistance of skilled personnel [5]. A review of sub-Saharan Africa demographic and health survey showed that only $13 \%$ of women who delivered at home received postnatal care within 2 days of birth [5]. The majority of health care providers across subSaharan Africa, including Eritrea, continue to advise mothers to come back to the facility for a first check-up after 6 weeks [6]. Despite these services and advice, maternal and neonatal mortality and morbidity in Eritrea were extremely high [7] . According to a WHO report, infant mortality rate was 36 deaths per 1000 live births and maternal mortality ratio was 501 per 100,000 live births in 2015 [7, 8]. In Eritrea, only $34 \%$ of the mothers who give birth were served by trained health workers [9]. Consequently, only $2 \%$ of women who had home deliveries receive postnatal care during the first 2 days of post-partum, and another 5 and $7 \%$ of such women had postnatal care within 5 to 41 days post-partum [10]. Hence, providing the needed services and advice might not always necessarily lead to achievement of the required goals.

Maternal and child health is one of the basic needs of a society for it is the cornerstone on which a health community and nation are built. Hence, the ministry of health of Eritrea has postnatal care program with sequence of activities that begins with clean delivery practice, followed by clean umblical cord care, thermal care, special care of low birth weight or preterm birth, early and exclusive breastfeeding, as well as immunization programs. However, postnatal care health education given to postpartum mothers in the maternity health facilities of Eritrea is not based on standard guidelines. On the other hand, while discharging the postpartum mothers from the hospital, list of common postnatal danger signs are given in a piece of paper to inform them that they have to approach a nearby health facility upon their appearance.

Postpartum mothers can pass the critical postpartum period successfully if they have knowledge regarding postnatal care [11]. A study conducted in Malawi on assessment of the knowledge and practice of postpartum mothers regarding postnatal care showed that almost all the participants were knowledgeable about some aspect of postnatal care [12]. However, other studies have shown women's insufficient knowledge on postnatal care $[13,14]$. As far as the researchers' knowledge is concerned, no published resource regarding maternal level of knowledge on postnatal care upon discharge in Eritrea exists. Therefore, this study is designed to determine maternal knowledge regarding PNC and to find out the socio-demographic determinants of knowledge scores on postnatal care among postpartum mothers.

\section{Methods}

\section{Study design and period}

Cross-sectional study design with quantitative approach was used to determine the knowledge of postnatal care among postpartum mothers during discharge in maternity hospitals in Asmara. The study was conducted in December, 2017.

\section{Study area and population}

The study was conducted in the health facilities which provide delivery service in Asmara. Asmara is the capital city of Eritrea, a country in the horn of Africa. This capital city is located $2325 \mathrm{~m}$ above sea level with a total area of $44.97 \mathrm{~km}^{2}$. According to 2017 Asmara municipality report, it has a population size of 416,367 . Maternity hospitals in the city are Orotta National Referral Maternity Hospital, Sembel Hospital, Edaga Hamus Community Hospital, and Betmekae Community Hospital. Postpartum mothers who delivered in these four hospitals during the study period constituted the study population.

\section{Participants}

Complete enumeration of the postpartum mothers was undertaken to determine the knowledge of postpartum mothers on postnatal care. This is because all subjects during the specified period of time can be recruited resulting to more accuracy, than that of samples. Hence, all health facilities (4 Hospitals) that render maternity 
services as well as all eligible postpartum mothers $(N=$ 334) who have given birth during the study period in the study area were considered but only 250 were finally included in the study.

\section{Variables}

The dependent variable in the study was the knowledge of postnatal care among mothers who had given birth. The selected determinants were age, marital status, religion, educational level, and occupation.

\section{Data collection tool and variable measurement}

A questionnaire was developed with reference to a guideline prepared by WHO on post-natal care of the mother and new born [1] and previous similar studies conducted in Kenya [15] and Tanzania [16]. After compiling the questionnaire, content validity was assessed using panel of experts from Ministry of Health and Asmara College of Health Sciences. On the other hand, the internal consistency of the tool was computed and found to be within the acceptable range (Richard's Kurdson $=0.75$ ). Then, the questionnaire was translated from English to Tigrigna, a language most familiar to Eritreans, by experienced researchers, linguists, and midwife experts.

The questionnaire was pre-tested among 30 postpartum mothers in Orotta National Referral Maternity Hospital 1 month before the study period. The interview was done face- to- face by five degree midwife nurses who can speak and understand the language. Pre-designed questions that were not easily understood by the interviewee were simplified after pre-testing the questionnaire. Furthermore, re-arrangement of the questions were made.

The modified questionnaire had two main parts, namely, socio-demographic characteristics and questions that assess knowledge on postnatal care. There were in total 17 questions (with 60 items each having one score) that were used to determine the knowledge on postnatal care encompassing two main components: maternal care, and baby care. Maternal care component consisted of concerns on maternal danger signs (15 items), infection prevention (9 items), bladder care (1 item), sexual activity starting time (1 item), proper nutrition (6 items), delay of menstruation by exclusive breast feeding ( 1 item), and contraceptive methods ( 4 items). On the other hand, the baby care component consisted of mechanism of keeping the baby warm (2 items), time of first new born baby bath (1 item), umbilical care (1 item), initiation of breast feeding (1 item), frequency of breast feeding per day (1 item), exclusive breast feeding (1 item), needs and purposes of vaccination ( 2 items), and baby danger signs (14 items). Every item was scored by assigning one of the following options: "correct" (score =1), and "wrong" (score = 0$)$. An overall score was obtained by adding the correct responses totaling to 60 . The scores indicate that with an increase in score, there is an increase of knowledge regarding postnatal care.

\section{Data entry and analysis}

After verification of the collected questionnaires by the researchers, the data was entered into CSPro (Census and Survey processing system) version 7.0 software package. The entered data was then exported to Statistical Package for Social Sciences (SPSS, version 22.0) for analysis. Frequency (percentage), mean (SD), or median (IQR) were used to describe the data, as appropriate. Normality of the knowledge score was assessed using Kolmogorov-Smirnov test. Independent samples t-test (variables with two categories) and one way ANOVA (variables with more than 2 categories) were used to find out the difference in the level of knowledge of post-natal across demographic variables. Bonferroni post hoc test was performed for the significant ANOVA results. Pvalues less than 0.05 were considered as significant.

\section{Operational definition}

Post-natal care refers to issues pertaining to the mother and the baby from birth up to 6 weeks [1].

Postpartum mothers are those mothers who have given birth in the health facility.

\section{Results}

Data collectors were able to approach 334 postpartum mothers in the four hospitals during the study period. However, 27 delivered by caesarean section and 307 by spontaneous vaginal delivery (SVD). In addition, 30 subjects were excluded because they cannot speak Tigrigna (native language), 13 had still birth, and 14 withdrew from the study to arrive at 250 subjects included in the analysis (Fig. 1).

\section{Background characteristics of the postpartum mothers}

An overview of the socio- demographic characteristics of 250 postpartum mothers during the study period on postnatal care is shown in Table 1.

Obstetrical and gynecological history of the mothers revealed that, 28.4, 23.2, 22.8, 8.4, and $17.2 \%$ were gravida one, two, three, four, and five and above respectively (Table 2). On the other hand, $33.2 \%$ of respondents were primi para, $24.4 \%$ were para two, $18.8 \%$ were para three, $8.4 \%$ were para four and $15.2 \%$ were para five and above. Only $37(14.8 \%)$ of respondents had history of abortion.

\section{Knowledge on maternal care}

The percentage distribution of postpartum mothers on maternal danger signs are shown in Fig. 2. The three most recognized maternal danger signs were heavy vaginal bleeding (83.2\%), severe head ache (38.4\%), and lower abdominal pain (32.0\%). 


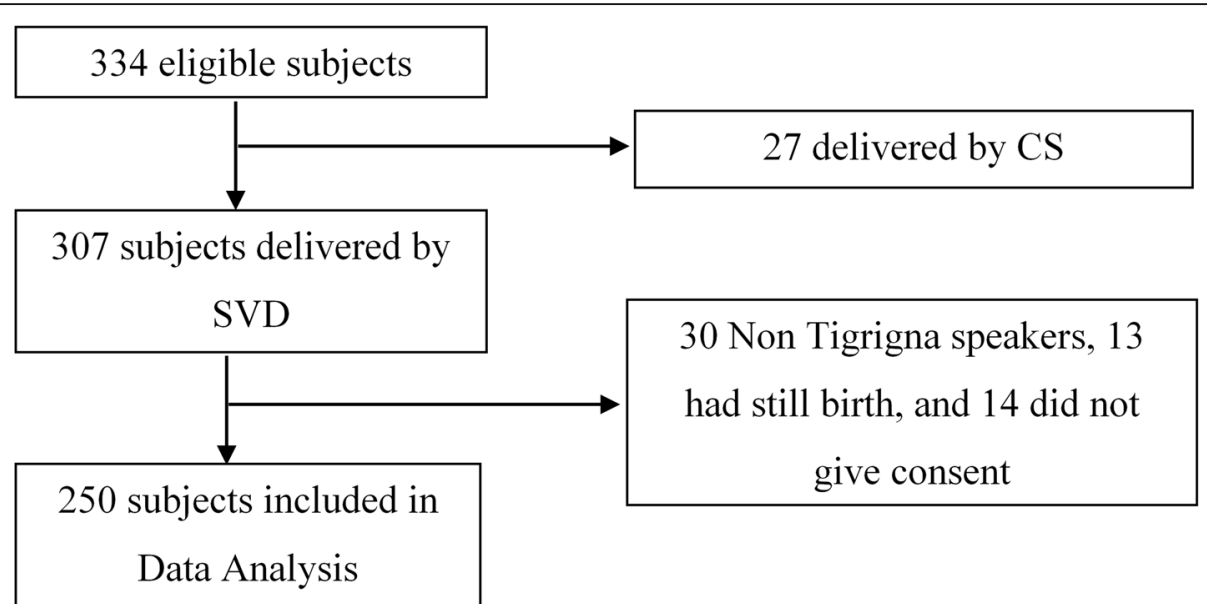

Fig. 1 Study participants that were eligible and finally included in the analyses

Table 1 Percentage distribution of postpartum mother by demographic characteristics $(n=250)$

\begin{tabular}{|c|c|c|}
\hline Variables & Frequency & Percentage \\
\hline \multicolumn{3}{|l|}{ Age (Years) } \\
\hline 17 to 25 & 93 & 37.2 \\
\hline 26 to 30 & 84 & 33.6 \\
\hline 31 to 42 & 73 & 29.2 \\
\hline \multicolumn{3}{|l|}{ Residence } \\
\hline Urban & 183 & 73.2 \\
\hline Rural & 67 & 26.8 \\
\hline \multicolumn{3}{|l|}{ Marital status } \\
\hline Married & 232 & 92.8 \\
\hline Living together & 5 & 2.0 \\
\hline Divorced & 2 & 0.8 \\
\hline Single & 11 & 4.4 \\
\hline \multicolumn{3}{|l|}{ Educational level } \\
\hline Junior and below & 73 & 29.2 \\
\hline Secondary & 143 & 57.2 \\
\hline Higher level & 34 & 13.6 \\
\hline \multicolumn{3}{|l|}{ Occupation $(n=244)$} \\
\hline Professionals & 47 & 19.3 \\
\hline House wife related & 197 & 80.7 \\
\hline \multicolumn{3}{|l|}{ Religion } \\
\hline Christian & 219 & 87.6 \\
\hline Muslim & 31 & 12.4 \\
\hline \multicolumn{3}{|l|}{ Ethnicity } \\
\hline Tigrigna & 234 & 93.6 \\
\hline Tigre/Saho/Afar & 16 & 6.4 \\
\hline
\end{tabular}

Almost all (96.0\%) of the respondents responded correctly on where to go if they note any danger signs (Table 3). Emptying the bladder every 2 hours, which is the correct response for frequency of urination, was mentioned only by $35(21.6 \%)$ of the postpartum mothers. The majority $(74.1 \%)$ of the respondents mentioned "if I felt to urinate". The minimum time for starting sexual intercourse was correctly responded by 114 (45.6\%). Six different nutrients which are needed to be taken during postpartum were presented to the postpartum mothers for identification. More than $80 \%$ of the postpartum mothers were able to identify food items rich in carbohydrates $(87.6 \%)$. Moreover, $81.6 \%$ replied high fluid intake. Food rich in proteins (75.2\%), vitamins

Table 2 Percentage distribution of postpartum mothers by obstetrical and gynecological history $(n=250)$

\begin{tabular}{lll}
\hline Variables & Frequency & Percentage \\
\hline Gravida & 71 & 28.4 \\
One & 58 & 23.2 \\
Two & 57 & 22.8 \\
Three & 21 & 8.4 \\
Four & 43 & 17.2 \\
Five or above & & \\
Para & 83 & 33.2 \\
One & 61 & 24.4 \\
Two & 47 & 18.8 \\
Three & 21 & 8.4 \\
Four & 38 & 15.2 \\
Five or above & & \\
Abortion & 213 & 85.2 \\
None & 37 & 14.8 \\
One or more & &
\end{tabular}




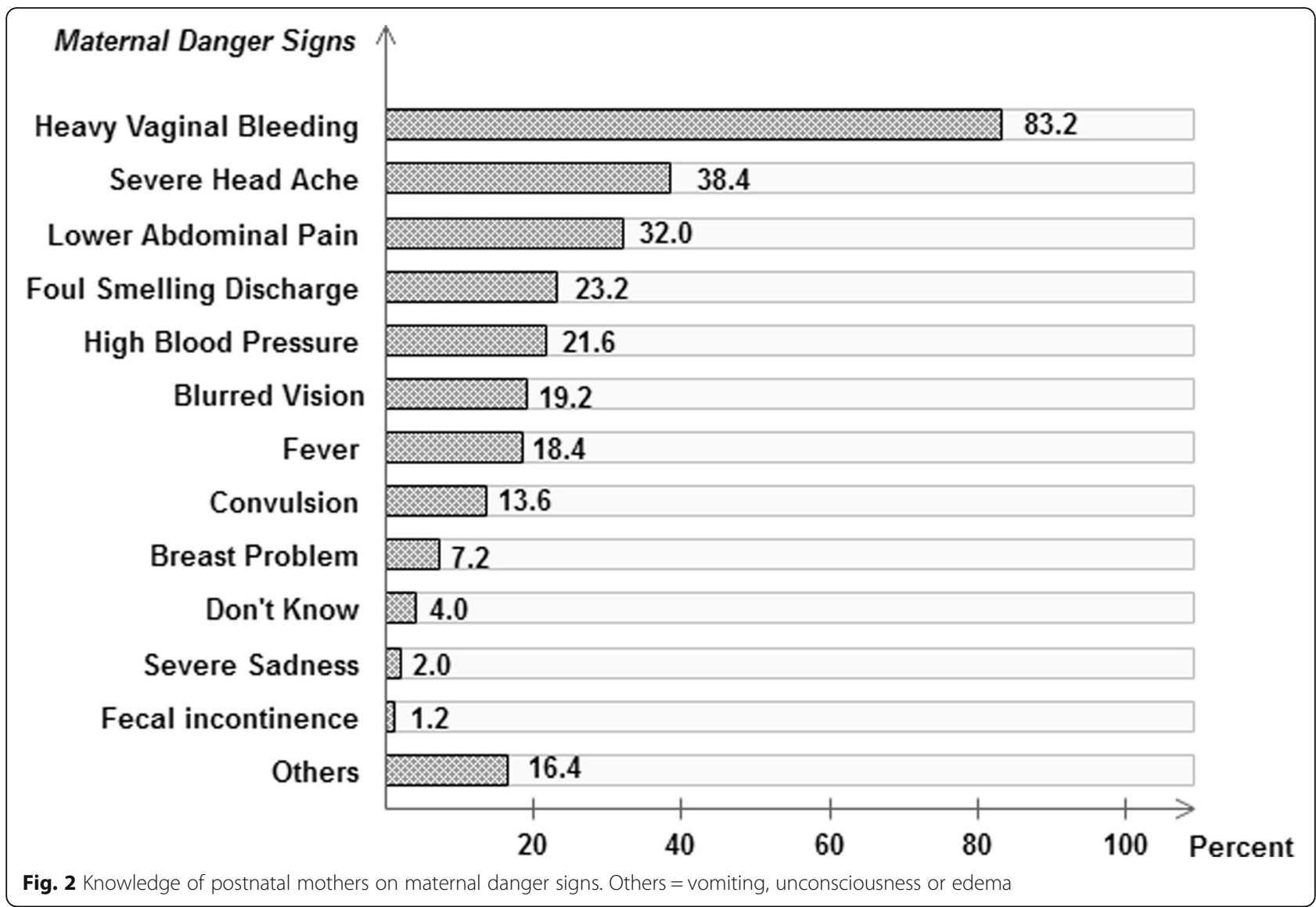

(67.6\%), fats (47.2\%), and minerals (45.6\%) were also mentioned. The percentages of women who responded delay in menstrual period as a result of giving exclusive breast feeding for 3 months, 6 months, 1 year, 2 years, and more than 2 years were 12.0, 26.4, 22.8, 17.6 and 21.2 respectively. More than nine tenth of postpartum mothers correctly identified injectable contraceptives $(92.7 \%)$ and oral contraceptive (91.5\%). The remaining had mentioned IUD (53.6\%), LAM (16.9\%) and other contraceptives $(46.8 \%)$ such as condom, Norplant or calendar method.

Nine different infection prevention methods were presented to the postnatal mothers for identification (Table 4). Wash perineum with warm water and some salt (70.0\%) and general body hygiene (69.6\%) were highly known infection prevention methods. The remaining seven infection prevention methods were known by less than half of the postpartum mothers. Among the least known infection prevention methods were hand washing after changing pads $(10.8 \%)$, hand washing after perineal hygiene (10.4\%), and hand washing before perineal hygiene (6.8\%).

\section{Knowledge on baby care}

Table 5 shows the percentage distribution of mothers by their knowledge on baby care. Knowledge on keeping the baby warm by wrapping the baby with cloth was almost universal (99.6\%). Few (6.8\%) also mentioned skin to skin contact. Correct response on the time at which first bath can be given for a new born baby was obtained from $67(26.8 \%)$ of the study participants. More than three fourths $(77.4 \%)$ of the women responded umbilical care should be simply keeping clean and dry. Most of the respondents $(88.40 \%)$ mentioned the correct answer on initiation of breast feeding after delivery which is within $30 \mathrm{~min}$. Almost three fourths (74.0\%) of the women correctly responded to the frequency need of breast feeding per day (eight per day); however, 10.4\% said 'if the baby cries'. The majority $(94.8 \%)$ of respondents correctly answered that duration of exclusive breast feeding needs to be for 6 months. Almost all respondents (99.2\%) correctly knew the needs of vaccine for a newborn baby. With regards to the purpose of vaccine, most of the respondents $(94.8 \%)$ mentioned 'to prevent disease' and 13 (5.2\%) did not know.

More than half of the respondents mentioned fever (58.8\%), severe vomiting (53.2\%), and difficulty in breathing (50.8\%) as baby danger signs (Table 6). Almost one third of the women were able to identify the inability to breast feed (32.8\%) and irritability (33.2\%). Less than one fourth of the respondents cited umbilical problems (14.0\%), abdominal 
Table 3 Percentage distribution of knowledge of mothers on maternal care attributes $(n=250)$

\begin{tabular}{|c|c|c|}
\hline Maternal care attributes & Frequency & Percentage \\
\hline \multicolumn{3}{|l|}{ Where to go after danger signs } \\
\hline Health facility & 240 & 96.0 \\
\hline Stay home or going to holy water & 10 & 4.0 \\
\hline \multicolumn{3}{|l|}{ Frequency of urination } \\
\hline Empty every $2 \mathrm{~h}$ & 35 & 21.6 \\
\hline I felt to urinate & 120 & 74.1 \\
\hline I don't know & 7 & 4.3 \\
\hline \multicolumn{3}{|l|}{ Return to sexual activity } \\
\hline After 42 days & 114 & 45.6 \\
\hline After 3 months & 74 & 29.6 \\
\hline After 6 months & 30 & 12.0 \\
\hline After 1 year & 15 & 6.0 \\
\hline I don't know & 17 & 6.8 \\
\hline \multicolumn{3}{|l|}{ Nutrients } \\
\hline Carbohydrate & 219 & 87.6 \\
\hline Fluids & 204 & 81.6 \\
\hline Proteins & 188 & 75.2 \\
\hline Vitamins & 169 & 68.0 \\
\hline Fats & 118 & 47.2 \\
\hline Minerals & 114 & 46.0 \\
\hline \multicolumn{3}{|l|}{ Delaying of period by exclusive breast feeding } \\
\hline 3 Months & 30 & 12.0 \\
\hline 6 Months & 66 & 26.4 \\
\hline 1 Year & 57 & 22.8 \\
\hline 2 Years & 44 & 17.6 \\
\hline More than two years & 53 & 21.1 \\
\hline \multicolumn{3}{|l|}{ Contraceptive method } \\
\hline Injectable & 230 & 92.7 \\
\hline Oral contraceptive pills & 227 & 91.5 \\
\hline Intrauterine device & 133 & 53.6 \\
\hline Lactational amenorrhea & 42 & 16.9 \\
\hline Condom, Norplant or Calendar method & 116 & 46.8 \\
\hline
\end{tabular}

distention (9.2\%), convulsion (8.4\%), lethargy (7.6\%), yellowness of eyes $(7.6 \%)$, eye problem $(6.8 \%)$, yellowness of palms (2.8\%), and yellowness of sole (1.6\%). However, 19 (7.6\%) of the respondents mentioned "I don't know" and 101 (40.4\%) responded vomiting/unconsciousness/ edema.

\section{Comparison of knowledge scores}

The results showed that the mean knowledge score was $24.89 / 60$ ( $\mathrm{SD}=5.66)$. Independent sample t-test has revealed that the categories in residence $(p<0.001)$ and ethnicity $(p=0.015)$ had shown significant difference in score of knowledge on postnatal care among postpartum
Table 4 Percentage of postpartum mothers on responses regarding infection prevention methods

\begin{tabular}{lll}
\hline Variables & Frequency & Percentage \\
\hline Hand washing before perineal hygiene & 17 & 6.8 \\
Hand washing after perineal hygiene & 26 & 10.4 \\
Hand washing after changing pads & 27 & 10.8 \\
Changing pads frequently & 91 & 36.4 \\
Changing pants frequently & 72 & 28.8 \\
General body hygiene & 174 & 69.6 \\
Wash with warm water and some salt & 175 & 70.0 \\
Washing after defecation & 89 & 35.6 \\
Washing after urination & 82 & 32.8 \\
\hline
\end{tabular}

mothers (Table 7). However, occupation $(p=0.210)$, religion $(p=0.476)$, number of abortion $(p=0.783)$ have not shown significant difference in score of knowledge on postnatal care among postpartum mothers.

Result from one way ANOVA (Table 8), revealed that there was significant difference in the average score of knowledge regarding post natal care among the postpartum mothers across different age groups $(p<0.001)$, marital status $(p=0.045)$, educational level $(p=0.014)$, gravidity $(p<0.001)$ and parity $(p<0.001)$. An increasing trend of knowledge score was observed with increase in age group $(p=0.001)$, educational level $(p=0.021)$, gravidity $(p<0.001)$ and parity $(p<0.001)$.

Bonferroni post-hoc comparison showed that the postnatal knowledge score among mothers aged 17-25 was significantly less than 26-30, and 31-42 years old. Moreover, significantly higher knowledge score was observed among married and divorced as compared to single and living together. Postpartum mothers who are junior or below were also found to have significantly lower postnatal knowledge score as compared to mothers who are secondary and higher level. No significant difference in knowledge score was observed among mothers who were gravidity two, three, four, and five or above, however, mothers of gravidity one had significantly less knowledge score than mothers of the aforementioned gravidity. Similar results with that of the gravidity were observed for parity.

\section{Discussion}

There are maternal and child health programs to safeguard maternal and child health in Eritrea. Nonetheless, maternal and neonatal mortality ratio still remain as high as 501/100,000 and 36/1000 live births, respectively, in the country [8]. Studies regarding utilization of maternal health services such as antenatal care and skilled delivery at birth are not infrequent; however, there still exists paucity of studies on knowledge regarding postnatal care in the country. One of the fundamental activities that needs to be instigated for the improvement of 
Table 5 Percentage distribution of knowledge of mothers regarding maternal care attributes $(n=250)$

\begin{tabular}{|c|c|c|}
\hline Baby care attributes & Frequency & Percentage \\
\hline \multicolumn{3}{|l|}{ Keeping the baby warm ${ }^{a}$} \\
\hline Skin to skin contact & 17 & 6.8 \\
\hline Wrap the baby with clothe & 249 & 99.6 \\
\hline \multicolumn{3}{|l|}{ First baby bath time } \\
\hline Immediately & 35 & 14.0 \\
\hline After $6 \mathrm{~h}$ & 27 & 10.8 \\
\hline After $24 \mathrm{~h}$ & 67 & 26.8 \\
\hline After 1 week & 17 & 6.8 \\
\hline After 3 days & 83 & 33.2 \\
\hline I don't know & 21 & 8.4 \\
\hline \multicolumn{3}{|l|}{ Umbilical care mechanism } \\
\hline Clean and dry & 147 & 77.4 \\
\hline Apply butter & 28 & 14.7 \\
\hline Apply cow dung & 1 & 0.5 \\
\hline Apply vaseline/oil & 79 & 31.7 \\
\hline I don't know & 14 & 7.4 \\
\hline \multicolumn{3}{|l|}{ Initiation of breast feeding } \\
\hline Within 30 min & 221 & 88.4 \\
\hline Within 1hour & 23 & 9.2 \\
\hline Within two to 3 hours & 5 & 2.0 \\
\hline Within 6 hours & 1 & 0.4 \\
\hline \multicolumn{3}{|l|}{ Frequency of breast feeding/day } \\
\hline At least 8 times & 185 & 74.0 \\
\hline If baby cries & 26 & 10.4 \\
\hline At least 4 to 6 times & 24 & 9.6 \\
\hline I don't know & 15 & 6.0 \\
\hline \multicolumn{3}{|c|}{ Duration of exclusive breast feeding } \\
\hline 3 Months & 7 & 2.8 \\
\hline 4 Months & 3 & 1.2 \\
\hline 6 Months & 237 & 94.8 \\
\hline 1 year & 3 & 1.2 \\
\hline \multicolumn{3}{|l|}{ Purpose of vaccination } \\
\hline To prevent disease & 237 & 94.8 \\
\hline I don't know & 13 & 5.2 \\
\hline
\end{tabular}

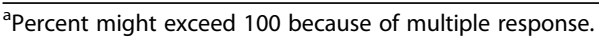

maternal and neonatal health is postnatal care because the majority of maternal and newborn death happen during this period [1]. Among others, one dimension of initiating postpartum care constitutes enhancing the knowledge of the mothers in order to enable them to properly handle themselves and the neonates in times of difficulty [17].

In this study, vaginal bleeding (83.2\%) was the most frequently mentioned danger sign during the postpartum
Table 6 Percentage distribution of knowledge of PN mothers on baby danger signs

\begin{tabular}{lll}
\hline Variables & Frequency & Percentage \\
\hline Yellowness of eyes & 19 & 7.6 \\
Yellowness of palms & 7 & 2.8 \\
Yellowness of soles & 4 & 1.6 \\
Umbilical problems & 35 & 14.0 \\
Eye problems & 17 & 6.8 \\
Unable to breast feed & 82 & 32.8 \\
Difficulty in breathing & 127 & 50.8 \\
Convulsion & 21 & 8.4 \\
Fever & 147 & 58.8 \\
Lethargic & 19 & 7.6 \\
Irritable & 83 & 33.2 \\
Abdominal distention & 23 & 9.2 \\
Sever vomiting & 133 & 53.2 \\
I do not know & 19 & 7.6 \\
Vomiting/unconsciousness/edema & 101 & 40.4 \\
\hline
\end{tabular}

period. This finding is similar to a research conducted in Ethiopia (89.2\%) [18] but lower than another study done in Nepal (98.47\%) [3]. When compared with the study conducted in Ethiopia, a similar result observed is foulsmelling vaginal discharge (23.3\%) as a danger sign [18]. However, in the Ethiopian study, relatively higher percentages of postnatal mothers identified severe headache (38.4\% Vs $23.1 \%)$, blurred vision (19.2\% Vs $8.9 \%)$, convulsion (13.6\% Vs $7.9 \%)$ and lower abdominal pain

Table 7 Comparison of knowledge of postpartum mothers on post natal care scores using t-test

\begin{tabular}{|c|c|c|c|}
\hline Variables & $\mathrm{M}(\mathrm{SD})$ & Diff. $^{a}(95 \mathrm{Cl})$ & $p$-value \\
\hline \multicolumn{4}{|l|}{ Residence } \\
\hline Urban & $25.69(5.79)$ & $2.97(1.38,4.57)$ & $<0.001$ \\
\hline Rural & $22.72(5.31)$ & & \\
\hline \multicolumn{4}{|l|}{ Occupation } \\
\hline Professional & $25.98(5.92)$ & $1.16(-0.66,2.98)$ & 0.210 \\
\hline House wife related & $24.82(5.64)$ & & \\
\hline \multicolumn{4}{|l|}{ Religion } \\
\hline Christian & $24.99(5.80)$ & $0.79(-1.40,2.99)$ & 0.476 \\
\hline Muslim & $24.19(5.88)$ & & \\
\hline \multicolumn{4}{|l|}{ Ethnic group } \\
\hline Tigrigna & $25.12(5.79)$ & $3.62(0.69,6.55)$ & 0.015 \\
\hline Tigre/Saho/Afar & $21.50(5.06)$ & & \\
\hline \multicolumn{4}{|l|}{ Number of abortion } \\
\hline None & $24.93(5.93)$ & $0.29(-1.76,2.33)$ & 0.783 \\
\hline One or more & $24.65(5.12)$ & & \\
\hline
\end{tabular}

${ }^{\mathrm{a} D i f f . ~ D i f f e r e n c e ~ i n ~ m e a n ~}$ 
Table 8 Comparison of knowledge on post natal care scores using ANOVA test

\begin{tabular}{|c|c|c|c|c|c|}
\hline Variable & $M(S D)$ & F-Value & $P$-value & $P$-value trend & Post Hoc Result ${ }^{a}$ \\
\hline \multicolumn{6}{|l|}{ Age group } \\
\hline $17-25$ & $22.88(5.73)$ & 9.68 & $<0.001$ & $<0.001$ & ${ }^{b} 17-25<26-30=31-42$ \\
\hline $26-30$ & $25.82(5.66)$ & & & & \\
\hline $31-42$ & $26.38(5.42)$ & & & & \\
\hline \multicolumn{6}{|l|}{ Marital status } \\
\hline Married & $25.16(5.70)$ & 2.72 & 0.045 & - & Married $>\mathrm{LT}=$ Single \\
\hline Living together & $19.80(6.46)$ & & & & Married = Divorced \\
\hline Divorced & $25.50(6.36)$ & & & & LT $<$ Divorced \\
\hline Single & $21.55(6.40)$ & & & & Divorced $>$ Single \\
\hline \multicolumn{6}{|l|}{ Educational level } \\
\hline Junior or below & $23.23(6.10)$ & 4.38 & 0.014 & 0.021 & $\mathrm{JB}<\mathrm{Secondary}=\mathrm{HL}$ \\
\hline Secondary & $25.65(5.69)$ & & & & \\
\hline Higher level & $25.26(5.01)$ & & & & \\
\hline \multicolumn{6}{|l|}{ Gravida } \\
\hline One & $22.03(5.83)$ & 7.42 & $<0.001$ & $<0.001$ & One $<$ Two, Three, Four, FA \\
\hline Two & $25.47(4.87)$ & & & & Two $=$ Three $=$ Four $=$ FA \\
\hline Three & $26.11(5.89)$ & & & & \\
\hline Four & $25.05(6.37)$ & & & & \\
\hline Five or above & $27.16(4.91)$ & & & & \\
\hline \multicolumn{6}{|l|}{ Para } \\
\hline One & $22.25(5.53)$ & 5.89 & $<0.001$ & $<0.001$ & One $<$ Two, Three, Four, FA \\
\hline Two & $25.89(5.29)$ & & & & Two $=$ Three $=$ Four $=$ FA \\
\hline Three & $25.89(6.03)$ & & & & \\
\hline Four & $25.38(5.80)$ & & & & \\
\hline Five or above & $27.55(4.91)$ & & & & \\
\hline
\end{tabular}

a Bonferroni post hoc test was performed, $L T$ Living together, JB Junior or below, $H L$ Higher level, $F A$ : Five or above

${ }^{b} 17-25<26-30=31-42$ : Knowledge score among 17-25 aged mothers is significantly less than those 26-30 and 31-42. Besides, knowledge score among $26-30$ aged mothers is not significantly different from 31 to 42

(32.0\% Vs 2.9\%) as danger signs as compared to this study [18]. This could be due to the long standing public health campaigns given in Eritrea that bleeding either during pregnancy or post-partum period puts the mother's health at danger.

In this study, the most identified infection prevention methods were washing perineum with warm water and some salt (70.0\%) and changing pads frequently (36.4\%). These findings are lower than those yielded in the study done in Nepal, in which $91.33 \%$ of PpM had prior knowledge of washing perineum with warm water and $83.16 \%$ changing pads frequently [3]. This discrepancy could be due to differences in the availability of health facilities and maternal training in the two study populations.

Around half $(45.6 \%)$ of the postpartum mothers were aware about the appropriate time for restarting sexual intercourse in this study. However, it is difficult to say whether the postpartum mothers' knowledge on this issue has been obtained through proper and methodical mechanisms, such as trainings and educational interventions, or from tradition. However, it is worth remembering that the scientific and traditional reason of commencing sexual intercourse after 6 weeks has the same ground. Lochia stays up to 6 weeks postpartum putting the mother at risk of postpartum infection and the pain as well as discomfort associated with the childbirth. Besides, the majority (87.6\%) of participants in this study were Christians, and specifically belonged to the Orthodox Christian religion, where women are traditionally obliged to abstain from sexual intercourse for a minimum of 40 days because they are considered as polluted (tainted) after childbirth.

Worldwide, unwanted pregnancy is a major cause of death in children less than 5 years of age and a death of pregnant women attempting abortion (650 per100,000 pregnant women) each year [11, 19]. Hence, a reliable contraceptive method is needed for mothers to avoid unwanted pregnancy as early as possible because menstruation period usually restarts at 3 to 9 weeks [11]. In the 
current study, knowledge of $\mathrm{PpM}$ on contraceptive methods shows that injectable contraceptives (92.7\%) and oral contraceptives (91.5\%) were highly mentioned. These findings are higher than the study done in Nepal that indicated injectable or Depo-Provera (78.0\%) followed by oral contraceptive pills $(74.0 \%)$ as the most common methods [20]. Therefore, it seems that maternal training and consultation about contraceptive methods in Eritrea is properly addressed and should be maintained.

In the present study, regarding knowledge of postpartum mothers on keeping baby warm after delivery, almost all participants $(99.6 \%)$ had a higher knowledge when compared to a study conducted in Nepal which showed that $82 \%$ had knowledge on wrapping the baby with warm clothes. However, in this study, lower level of knowledge (6.8\%) was scored on keeping baby warm by skin to skin contact than the study done in Nepal (58\%) [21].

Almost one-fourth of the PpM knew the correct answer for 'when to give a bath to a newly born baby' which is similar to a study conducted in India (30\%) [22]. Around three-fourths of the postpartum mothers in the current study responded that umbilical cord is taken care of by simply keeping the umbilicus clean and dry. However, the study in Nepal showed that 59\% were knowledgeable about keeping the umbilicus clean and dry [21]. The culture-driven beliefs and practices have also led $14.7 \%$ of the postpartum mothers in this study to indicate that butter is to be applied for umbilical care.

Health education provision by health workers at the health facilities and through the mass media could be the possible reason behind the correct knowledge $(88.4 \%)$ of postpartum mothers regarding initiation of breastfeeding within $30 \mathrm{~min}$. However, in a study done in Nepal, the percentage of mothers who mentioned that breastfeeding needs to be initiated within 1 hour was 48\% [21]. Fever, as a new born danger sign, was identified by only $58.8 \%$ of the postpartum mothers in this study. This can be said to be relatively lower than the findings in Ethiopia (76.6\%) [18] and Kenya (74.9\%) [15]. Moreover, the difficulty of breathing as a baby danger sign was mentioned by almost half $(50.8 \%)$ of the postpartum mothers. The same danger sign, was known by $46.6 \%$ of the participants in Kenyan study [15], whereas the current study finding was higher than that of Ethiopian study (19.7\%) [14].

Results regarding new-born danger signs on umbilical cord problem $(14,5.4,35 \%)$, convulsion $(8.4,19.3,15 \%)$, and eye problem $(6.8,16.7,21 \%)$ were not similar in this study, Ethiopia, and Nepal respectively [14, 21]. Yellow palm as baby danger sign was the most highly unidentified $(97.2 \%)$ by the postpartum mothers in this study, which is similar to the findings in Ghana (93.6\%) [23]. However, inconsistent results on jaundice as baby danger sign were obtained in a study done in Nepal (21\%) [21].
Comparison of the knowledge scores by categories of residence has revealed that urban residents had significantly greater knowledge score than the rural residents. The main reason for the difference could be the regularity in health education in urban places. Another possible reason could be the easy access to transport in urban places to go to the place where health education is offered. The lower level of knowledge among mothers who were in the age group 17 to 25 , or primipara could be due to practical lessons that multipara mothers gain each time birth occurs.

\section{Limitations of the study}

The use of a cross sectional design in this study did not allow for causal relationships to be established, thus the reasons why postpartum mothers reported certain maternal and baby care more so than others is not known.

\section{Conclusion}

The average knowledge score on PNC of the postpartum mothers was low for it was below half of the overall score. Moreover, significant association between the mothers' knowledge and their age group, residence, educational background, and parity was observed. Therefore, special attention should be given to mothers living in rural areas, junior or below in educational level, single or living together, primigravida/primipara, ethnic groups other than Tigrigna, and those between 17 to 25 years of age to improve PNC knowledge.

\section{Abbreviations \\ ANOVA: Analysis Of Variance; CS: Cesarean Section; IQR: Interquartile range; PNC: Post Natal Care; PpM: Postpartum Mothers; SD: Standard Deviation; SPSS: Statistical Package for Social Sciences; SVD: Spontaneous vaginal delivery; WHO: World Health Organization}

\section{Acknowledgments}

We thank the staffs at the study areas for their assistance and collaboration. We would like also to express our heartfelt appreciation to the mothers who have participated in this study.

\section{Authors' contributions}

Each author contributed substantially to manuscript. GGB conceived, designed, wrote, analyzed and interpreted the manuscript. AG, BY, KH, ST, and SG collected the data and analyzed and wrote the first draft of the paper. EHT designed, analyzed and critically revised the manuscript for important intellectual content the final paper. All authors have read the manuscript for publication. All authors read and approved the final manuscript.

\section{Funding}

No funds were available.

\section{Availability of data and materials}

Data set is available in electronic form which can be accessed upon a reasonable request from the corresponding author.

\section{Ethics approval and consent to participate}

Ethical approval was obtained from Asmara College of Health Sciences with a ref. No.: 019/09/17 and Eritrean Ministry of Health research and ethics committee with a ref. No:: 002/10/17. The participants' rights were protected by ensuring voluntary participation and supported by written consent; after 
explaining the purpose, nature, potential benefits and risks of the study and data collection techniques. The anonymity and confidentiality of the participants were assured and treated as strictly confidential. Furthermore, they were given a full right to drop from participating in the study.

\section{Consent for publication}

Not applicable.

\section{Competing interests}

The authors declare that they have no competing interests.

\section{Author details}

'Department of Nursing, Orotta College of Medicine and Health Sciences, Asmara, Eritrea. ${ }^{2}$ Department of Statistics, Biostatistics and Epidemiology Unit, College of Science, Eritrea Institute of Technology, Mai-Nefhi, Eritrea.

Received: 21 August 2019 Accepted: 23 December 2019

Published online: 06 January 2020

\section{References}

1. World Health Organization. WHO recommendations on postnatal care of the mother and newborn. Geneva: World Health Organization; 2014.

2. FCM, Early Postpartum Care of the Mother and Infant and Transition to the Community.

3. Timilsina S, Dhakal R. Knowledge on postnatal care among postnatal mothers. Saudi J Med Pharm Pci. 2015:1:87-92.

4. Shahraki $A D$, et al. World health report: make every mother and child count J Med Sci. 2005;7(8):1066-74

5. WHO. Postnatal care for mothers and newborns. Geneva: Highlights from the World Health Organization 2013 guidelines. RHR/15.05; 2015.

6. Warren C, et al. Postnatal care opportunities for Africa newborn. Oxford: Oxford University; 2005.

7. Alkema $\mathrm{L}$, et al. Global, regional, and national levels and trends in maternal mortality between 1990 and 2015, with scenario-based projections to 2030: a systematic analysis by the UN maternal mortality estimation inter-agency group. Lancet. 2016;387(10017):462-74.

8. World Health Organization. Trends in Maternal Mortality: 1990 to 2015. Geneva: World Health Organization; 2015.

9. NSO. Eritrea Population and Health Survey 2010. Asmara: National statistics Offce and Fafo Institute for Appled; 2010.

10. Araya W, et al. Overview of maternal mortality in Eritrea, sub-Saharan Africa. Afr J Midwifery Womens Health. 2012:6(4):171-5.

11. Mirzaee K, Taghi Shakeri M. Maternal knowledge on postpartum care in healthcare centers of Mashhad, Iran in 2013. J Midwifery Reprod Health. 2015;3(4):456-64.

12. Sarkar J, Konwar G, Das LK. Assessment of knowledge and practice regarding postnatal exercises among mothers admitted in postnatal ward in Gauhati medical college and hospital. Obstet Gynaecol Barpeta. 2014;1(1):52-6.

13. Mohamadirizi S, Bahrami B, Moradi F. Comparison of the effect of electronic education and pamphlet on the knowledge of women about their post partum hygiene. J Nurs Educ. 2015;3(4):29-36.

14. Feyisso M, Addisu Y. Prabhanja danger signs of neonatal and postnatal illness and health seeking. J Curr Res. 2016;8(01):25466-71.

15. Kibaru EG, Otara AM. Knowledge of neonatal danger signs among mothers attending well baby clinic in Nakuru Central District, Kenya: cross sectional descriptive study. BMC Res Notes. 2016;9(1):481.

16. Mwilike $B$, et al. Knowledge of danger signs during pregnancy and subsequent healthcare seeking actions among women in urban Tanzania: a cross-sectional study. BMC Pregnancy Childbirth. 2018:18(1):4

17. Fahey JO, Shenassa E. Understanding and meeting the needs of women in the postpartum period: the perinatal maternal health promotion model. J Midwifery Womens Health. 2013;58(6):613-21.

18. Bililign N, Mulatu T. Knowledge of obstetric danger signs and associated factors among reproductive age women in Raya kobo district of Ethiopia: a community based cross-sectional study. BMC Pregnancy Childbirth. 2017; 17(1):70

19. Zamani F, Rajabi Z. Unwanted pregnancy in rural women of Najafabad. KAUMS J (FEYZ). 2005:9(1):56-61.

20. Tuladhar $H$, Marahatta R. Awareness and practice of family planning methods in women attending gyne OPD at Nepal medical college teaching hospital. Nepal Med Coll J. 2008;10(3):184-91.
21. Shrestha T, Bhattarai SG, Silwal K. Knowledge and practice of postnatal mother in newborn care. J Nepal Med Assoc. 2013:52(190):372-7.

22. Rama R, Gopalakrishnan S, Udayshankar P. Assessment of knowledge regarding new-born care among mothers in Kancheepuram district, Tamil Nadu. Int J Commun Med Public Health. 2017;1(1):58-63.

23. Kuganab-Lem R, Yidana A. Exploring women knowledge of newborn danger signs: a case of mothers with under five children. Public Health Res. 2014;4(5):195-202.

\section{Publisher's Note}

Springer Nature remains neutral with regard to jurisdictional claims in published maps and institutional affiliations.
Ready to submit your research? Choose BMC and benefit from:

- fast, convenient online submission

- thorough peer review by experienced researchers in your field

- rapid publication on acceptance

- support for research data, including large and complex data types

- gold Open Access which fosters wider collaboration and increased citations

- maximum visibility for your research: over $100 \mathrm{M}$ website views per year

At BMC, research is always in progress.

Learn more biomedcentral.com/submissions 